\title{
AVALIAÇÃO DE DIFERENTES FONTES E NÍVEIS DE SELÊNIO PARA FRANGOS DE CORTE EM DIFERENTES TEMPERATURAS
}

\author{
(Evaluation of different sources and selenium levels for broilers at different temperatures)
}

DAHLKE, F.'; GONZALES, E. ${ }^{2}$; FURLAN. R.L. ${ }^{3}$; GADELHA, A.C. ${ }^{4}$ MAIORKA, A. ${ }^{1}$; ALMEIDA, J.G. ${ }^{5}$

'Departamento de Zootecnia, UFPR, Curitiba, PR; ${ }^{2}$ Faculdade de Medicina Veterinária e Zootecnia, Unesp/Botucatu, SP; ${ }^{3}$ Faculdade de Ciências Agrárias e Veterinárias, Unesp, Jaboticabal, SP; ${ }^{4}$ Departamento de Zootecnia, Universidade Federal do Ceará, Fortaleza, CE; ${ }^{5}$ Departamento de Zootecnia, Universidade Federal do Rio Grande do Sul, RS.

RESUMO - Foram avaliados os parâmetros zootécnicos de frangos de corte alimentados com dietas suplementadas com diferentes fontes e níveis de selênio (Se), criados sob diferentes condições de temperatura. Foram utilizados 720 pintainhos, Cobb-500, em um delineamento inteiramente ao acaso em esquema fatorial $3 \times 2 \times 2$ ( 3 temperaturas ambiente: termoneutra, fria e quente; 2 níveis de suplementação de Se: nível A e B; e 2 fontes de Se: orgânica e inorgânica), com 4 repetições de 15 aves. Os níveis $A$ e $B$ de Se na ração inicial (1 a 21 dias) e de crescimento (22 a 42 dias) foram 0,15 e $0,30 \mathrm{mg} / \mathrm{kg}$ e 0,20 e $0,40 \mathrm{mg} / \mathrm{kg}$, respectivamente. Selenometionina foi usada como fonte de Se orgânico e selenito de sódio, inorgânico. Os frangos criados em condições de estresse por calor apresentaram uma redução no consumo de ração e ganho de peso. Não houve influência da suplementação das diferentes fontes ou níveis de Se no ganho de peso, consumo de ração ou conversão alimentar. Os resultados são indicativos de que a suplementação de Se não melhora o desempenho de frangos de corte criados em condições de temperatura adversa.

Palavras-chave: desempenho, frangos de corte, selênio, temperatura ambiente.

ABSTRACT - This study evaluated the effect of selenium (Se) diet supplementation on productive parameters of broiler chickens raised under different temperatures, in a complete randomized design with $3 \times 2 \times 2$ factorial schedule ( 3 environmental temperatures: cold, neutral and hot; 2 levels and two Se sources) with 4 repetitions of 15 birds. The levels of Se in rations for initial and growth phases were: 0.15 and $0.30 \mathrm{mg} / \mathrm{kg}$; and 0.20 and $0.40 \mathrm{mg} / \mathrm{kg}$, respectively. The organic and inorganic sources of selenium were selenomethionine and sodium selenite, respectively. There were a reducing in broiler feed intake and weight gain of the broilers housed in the hot temperature. Selenium levels or sources did not affect the weight gain, feed intake or feed conversion. It was concluded that Se supplementation did no affect the broiler performance, housed under adverse environmental temperature.

Key-words: broiler, environmental temperature, performance, selenium. 


\section{Introdução}

Sabe-se que o selênio (Se) está envolvido nos mais diversos processos metabólicos e bioquímicos no organismo animal, como na síntese dos aminoácidos contendo enxofre, principalmente na eficiência da transsulfuração, que resulta na formação das cisteína a partir da metionina (HALPIN e BAKER, 1984), importante no crescimento de diversas estruturas corporais. O Se também atua no metabolismo dos hormônios da tireóide principalmente no metabolismo do hormônio 3,5,3' -triiodotironina $\left(T_{3}\right)$ que é produzido pela deiodinação da Tiroxina $\left(T_{4}\right)$ em tecidos e órgãos periféricos através da seleno-proteína iodotironina deiodinase tipo I (5'ID) (BECKETT et al., 1992). O hormônio $T_{3}$ atua sobre o crescimento do animal através do turnover protéico (HAYASHI, 1993). Deficiência de Se poderia afetar este turnover e, conseqüentemente, o crescimento animal, pela redução da síntese de $T_{3}$. JIANHUA et al. (2000) testaram esta possibilidade empregando níveis crescentes de Se e verificaram que o aumento do nível deste mineral na dieta determinou um aumento na concentração de 5'deiodinase e do hormônio T3, com concomitante melhora no desenvolvimento das aves.

A alta temperatura ambiente e umidade relativa do ar são um dos principais fatores que prejudicam as características de interesse zootécnico dos frangos de corte, o que tem feito aumentar o interesse por práticas nutricionais e ambientais que possam amenizar seus efeitos (FARIA FILHO, 2003). Da mesma forma, os hormônios $T_{3}$ e $T_{4}$ podem ser alterados pela temperatura ambiente. Exposição da ave a temperaturas baixas promove aumento dos níveis sangüíneos de $T_{3}$ com efeito inverso em temperaturas elevadas (HADDAD e MASHALY, 1989). Altos níveis destes hormônios promovem maiores consumos de oxigênio, ativam a sintese e metabolismo de proteínas, carboidratos e lipídios (HWANG-BO et al., 1990). Como existe um aumento na atividade da 5'deiodinase, enzima ativadora do hormônio $\mathrm{T}_{3}$, como conseqüência do aumento da ingestão de Se (JIANHUA et al.,2000), acredita-se que alterações do crescimento podem ser promovidas com a suplementação deste mineral.

Desta forma, o presente estudo objetivou avaliar o efeito da suplementação dietética de selênio sobre os parâmetros produtivos (consumo de ração, ganho de peso e conversão alimentar) de frangos de corte submetidos a estresse por calor e frio.

\section{Material e Métodos}

Foram utilizados 720 frangos de corte, machos, da linhagem híbrida comercial Cobb$500 \AA$. Do primeiro ao quarto dia as aves criadas em temperatura de $32^{\circ} \mathrm{C}$ (termoneutra). A partir do $4^{\circ}$ dia de idade as aves foram distribuídas em câmaras climáticas, sob três condições de temperatura: termoneutra, (de acordo com a idade das aves); quente e fria (TABELA 1).

As aves foram distribuídas em boxes, com dimensões $2,50 \times 1,10 \mathrm{~m}$, segundo um delineamento inteiramente ao acaso, em um modelo fatorial $2 \times 2 \times 3$ (níveis de selênio, fontes de selênio e temperaturas ambiente) com 4 repetições de 15 aves cada.

As rações, a base de milho e farelo de soja, foram isonutritivas (TABELA 2), e para a obtenção dos níveis desejados de selênio, foram suplementados à dieta basal $0,15 \mathrm{mg} e$ $0,3 \mathrm{mg}$ de Se por $\mathrm{kg}$ de ração, na fase inicial; $0,2 \mathrm{mg}$ e 0,4 $\mathrm{mg}$ de Se/kg de ração na fase de crescimento. $\mathrm{O}$ Se foi adicionado à dieta na forma inorgânica (selenito de sódio - $\mathrm{Na}_{2} \mathrm{SeO}_{3}$ ) ou orgânica (Selenometionina - SO). As dietas foram assim constituídas: Ração1 (R1) - 0,15 $\mathrm{mg}$ de Se fornecido através de selenito de sódio, Ração 2 (R2) - 0,30 mg de Se fornecido através de selenito de sódio, Ração 3 (R3) $0,15 \mathrm{mg}$ de Se fornecido através de Se orgânico e Ração 4 (R4) - 0,30 mg de Se fornecido através de Se orgânico, para a fase inicial. Para a fase de crescimento as dietas foram: Ração 5 (R5) - 0,20 mg de Se fornecido através de selenito de sódio, Ração 6 (R6) - 0,40 mg de Se fornecido através de selenito de sódio, Ração 7 (R7) - 0,20 mg de Se fornecido na forma de Se orgânico e Ração 8 (R8) - 0,40 $\mathrm{mg}$ de Se fornecido na forma de Se orgânico (TABELA 2).

Para avaliar as características de desempenho, toda a ração fornecida, a sobra de ração e as aves foram pesadas ao 
Avaliação de diferentes fontes e níveis de selênio para frangos de corte em diferentes temperaturas

alojamento, 21 e 42 dias de idade. Foram estudados consumo de Ração (CR), ganho de peso corporal (GP) e conversão alimentar (CA) para o período de 1 a 21 e 1 a 42 dias de idade.

Os dados obtidos foram submetidos à análise de variância através do procedimento General Linear Model (GLM) do programa SAS ${ }^{\circledR}$ (SAS Institute, 2002) e em caso de diferença estatística significativa as médias foram comparadas pelo teste de tukey à $5 \%$ de probabilidade.

TABELA 1 - TEMPERATURA MÉDIA DAS CÂMARAS CLIMÁTICAS DURANTE O PERÍODO EXPERIMENTAL.

\begin{tabular}{lccc}
\hline \multirow{2}{*}{ Idade (dias) } & \multicolumn{3}{c}{ Temperatura ambiente $\left({ }^{\circ} \mathrm{C}\right)$} \\
\cline { 2 - 4 } & Fria & Termoneutra & Quente \\
\hline $1-14$ & 24,8 & 31,0 & 35,0 \\
$15-21$ & 18,2 & 30,4 & 33,6 \\
$21-42$ & 15,1 & 25,3 & 30,7 \\
\hline
\end{tabular}

\section{Resultados}

Os resultados para consumo de ração (CR), ganho de peso corporal (GP) e conversão alimentar (CA) são apresentados na TABELA 3. Não ocorreram interações entre os fatores para todas as características de desempenho estudadas.

Os frangos de corte criados em ambiente quente apresentaram menor consumo de ração e menor ganho do peso em relação aos frangos criados no frio ou termoneutralidade, aos 21 e 42 dias de idade. Houve uma piora na conversão alimentar, aos 21 dias, nos frangos criados fora da termoneutralidade.

A utilização de Selenito de Sódio ou Selênio Orgânico não alterou as variáveis de desempenho em nenhuma das idades estudadas $(P>0,05)$. Da mesma forma, a utilização de níveis altos ou baixos de selênio não influenciou os parâmetros zootécnicos avaliados aos 21 e 42 dias de idade.

\section{Discussão}

A redução do consumo foi acompanhada por um menor ganho de peso nas aves criadas em ambiente quente, na fase de crescimento e no período total de criação. Em altas temperaturas, a troca respiratória é o mecanismo mais eficiente para dissipação de calor, sendo até seis vezes mais eficientes que os demais mecanismos (MELTZER, 1987). De 19 a $30^{\circ} \mathrm{C}$, a taxa respiratória aumenta quatro vezes e conseqüentemente ocorrem perdas energéticas em função desta maior atividade (LEESON,1986). Da mesma forma com que a homeotermina é mantida com um gasto mínimo de energia, em condição de termoneutralidade (FURLAN e MACARI, 2002), existe uma maior exigência de energia metabolizável para a mantença fora da faixa termoneutra (LONGO, 2000). A redução na ingestão de alimento aliada a uma pior eficiência na utilização da energia metabolizável na condição de hipertermia (FARIA FILHO, 2003), explicam o menor ganho de peso dos frangos submetidos ao calor.

A utilização de selenito de sódio ou selênio orgânico, nos níveis estudados, não alterou as variáveis de desempenho em nenhuma das idades estudadas. Segundo COMBS (1981) as recomendações para o nível dietético de Se para frangos de corte, listadas no NRC (1994) de $0,15 \mathrm{mg} / \mathrm{kg}$ - são insuficientes para atender as necessidades das aves até os 21 dias de idade. JIANHUA et al. (2000) também sugeriram a utilização de níveis maiores, de $0,3 \mathrm{mg} / \mathrm{kg}$, para um maior desempenho. No entanto, os níveis de selênio utilizados neste estudo $(0,15$ até $0,4 \mathrm{mg} / \mathrm{kg}$ ) não alteraram os parâmetros zootécnicos. MOREIRA et al. (2001) também não encontraram diferença para consumo, ganho de peso ou conversão alimentar, estudando a suplementação em níveis crescentes de Se na dieta $(0,15 ; 0,45 ; 0,75$; 1,05 e 1,35 $\mathrm{mg}$ de Se/kg de ração). 
DAHLKE, F. et al.

TABELA 2 - COMPOSIÇÃO (\%) DAS DIETAS EXPERIMENTAIS NA FASE INICIAL (1- 21DIAS DE IDADE) E FASE DE CRESCIMENTO (22-42 DIAS DE IDADE).

\begin{tabular}{|c|c|c|c|c|c|c|c|c|}
\hline & \multicolumn{4}{|c|}{$1-21$ dias } & \multicolumn{4}{|c|}{$22-42$ dias } \\
\hline Ingredientes (\%) & $\mathrm{R} 1^{3}$ & R2 & R3 & $\mathrm{R} 4$ & R5 & R6 & R7 & R8 \\
\hline Milho & 51,00 & 51,00 & 51,00 & 51,00 & 56,60 & 56,60 & 56,60 & 56,60 \\
\hline Farelo de soja & 40,43 & 40,43 & 40,43 & 40,43 & 34,68 & 34,68 & 34,68 & 34,68 \\
\hline Óleo de soja & 4,59 & 4,59 & 4,59 & 4,59 & 5,10 & 5,10 & 5,10 & 5,10 \\
\hline Fosfato bicálcico & 1,74 & 1,74 & 1,74 & 1,74 & 1,72 & 1,72 & 1,72 & 1,72 \\
\hline Calcário & 1,40 & 1,40 & 1,40 & 1,40 & 1,22 & 1,22 & 1,22 & 1,22 \\
\hline Sal comum & 0,40 & 0,40 & 0,40 & 0,40 & 0,32 & 0,32 & 0,32 & 0,32 \\
\hline Suplemento vit. /mineral ${ }^{2}$ & 0,10 & 0,10 & 0,10 & 0,10 & 0,10 & 0,10 & 0,10 & 0,10 \\
\hline DL-metionina & 0,29 & 0,29 & 0,29 & 0,29 & 0,21 & 0,21 & 0,21 & 0,21 \\
\hline Butil Hidroxitolueno & 0,03 & 0,03 & 0,03 & 0,03 & 0,03 & 0,03 & 0,03 & 0,03 \\
\hline Porção variável ${ }^{1}$ & 0,02 & 0,02 & 0,02 & 0,02 & 0,02 & 0,02 & 0,02 & 0,02 \\
\hline Composição calculada & \multicolumn{4}{|c|}{$1-21$ dias } & \multicolumn{4}{|c|}{$22-42$ dias } \\
\hline Energia Metabolizável (kcal/kg) & \multicolumn{4}{|c|}{3050} & \multicolumn{4}{|c|}{3150} \\
\hline Proteína Bruta (\%) & \multicolumn{4}{|c|}{22,00} & \multicolumn{4}{|c|}{20,00} \\
\hline Cálcio (\%) & \multirow{2}{*}{\multicolumn{4}{|c|}{$\begin{array}{l}1,00 \\
0,44\end{array}$}} & \multicolumn{4}{|c|}{0,90} \\
\hline Fósforo Disponível (\%) & \multirow{2}{*}{\multicolumn{4}{|c|}{$\begin{array}{l}0,44 \\
0,20\end{array}$}} & \multicolumn{4}{|c|}{0,43} \\
\hline Sódio (\%) & & & & & \multicolumn{4}{|c|}{0,20} \\
\hline Metionina (\%) & \multicolumn{4}{|c|}{0,57} & \multicolumn{4}{|c|}{0,45} \\
\hline Metionina + Cistina (\%) & \multicolumn{4}{|c|}{0,95} & \multicolumn{4}{|c|}{0,82} \\
\hline Lisina (\%) & \multicolumn{4}{|c|}{1,20} & \multirow{2}{*}{\multicolumn{4}{|c|}{$\begin{array}{l}1,00 \\
0,83\end{array}$}} \\
\hline Potássio (\%) & \multicolumn{4}{|c|}{0,90} & & & & \\
\hline Cloro (\%) & \multicolumn{4}{|c|}{0,35} & \multicolumn{4}{|c|}{0,35} \\
\hline
\end{tabular}

1. A porção variável foi constituída de selenito de sódio (selênio inorgânico) e selenometionina (selênio orgânico) e inerte, a fim de se obter os níveis de 0,15 e 0,30 $\mathrm{mg}$ de Se/kg de ração de 1 a 21 dias ; 0,20 $\mathrm{mg}$ e 0,40 $\mathrm{mg} / \mathrm{kg}$ de ração de 21 a 42 dias de idade.

2. Suplemento Mineral e Vitamínico: Cada quilograma do produto contém: vitamina $A$ 90.000Ul; vitamina $E$ $20 \mathrm{mg}$; vitamina K 2,5 mg; vitamina B1 2,0 mg; vitamina B2 6,6 mg; vitamina B12 20,0 mcg; ácido fólico 0,1 mg; ácido pantotênico 10,0 m; niacina $100,0 \mathrm{mg}$, biotina $0,15 \mathrm{mg}$; colina $1,54 \mathrm{~g}$; antioxidante $0,125 \mathrm{mg}$; iodo $0,35 \mathrm{mg}$; cobre $10,0 \mathrm{mg}$; ferro $50,0 \mathrm{mg}$; manganês $88,0 \mathrm{mg}$; zinco $0,1 \mathrm{mg}$; enxofre $0,72 \mathrm{~g}$; bacitracina de zinco 0,03 ; Coxixtac 0,05 .

\section{Constituições das rações}

Ração 1 (R1) - 0,15 mg de Se fornecido através de selenito de sódio,

Ração 2 (R2) - 0,30 mg de Se fornecido através de selenito de sódio,

Ração 3 (R3) - 0,15 mg de Se fornecido através de Se orgânico,

Ração 4 (R4) - 0,30 mg de Se fornecido através de Se orgânico, para a fase inicial.

Ração 5 (R5) - 0,20 mg de Se fornecido através de selenito de sódio,

Ração 6 (R6) - 0,40 mg de Se fornecido através de selenito de sódio,

Ração 7 (R7) - 0,20 mg de Se fornecido na forma de Se orgânico

Ração 8 (R8) - 0,40 mg de Se fornecido na forma de Se orgânico, para a fase de crescimento

\section{Conclusões}

A temperatura de criação é um componente de suma importância no desempenho de frangos, afetando diretamente os parâmetros produtivos, como ganho de peso e consumo de ração.

Não houve evidências suficientes para concluir que a suplementação de selênio, em níveis de até $0,4 \mathrm{mg} / \mathrm{kg}$ de ração melhore o desempenho, independente da condição térmica do meio. 
Avaliação de diferentes fontes e níveis de selênio para frangos de corte em diferentes temperaturas

TABELA 3 - CONSUMO DE RAÇÃO (CR), GANHO DE PESO (GP) E CONVERSÃO ALIMENTAR (CA) DE FRANGOS DE CORTE DE 1 A 21 E 1 A 42 DIAS DE IDADE ALIMENTADOS COM DIETAS SUPLEMENTADAS COM DIFERENTES NÍVEIS DE SELÊNIO, NA FORMA ORGÂNICA E INORGÂNICA, CRIADOS EM DIFERENTES TEMPERATURAS.

\begin{tabular}{|c|c|c|c|c|c|c|}
\hline \multirow[b]{2}{*}{ Fatores } & \multicolumn{3}{|c|}{21 dias } & \multicolumn{3}{|c|}{42 dias } \\
\hline & $C R(g)$ & GP (g) & $\mathrm{CA}(\mathrm{g} / \mathrm{g})$ & CR (g) & $\mathrm{GP}(\mathrm{g})$ & $\mathrm{CA}(g / g)$ \\
\hline \multicolumn{7}{|c|}{ Temperatura ambiente } \\
\hline Fria & $1083 a$ & $697 \mathrm{a}$ & $1,57 a$ & $4247 a$ & $2313 a$ & 1,84 \\
\hline Termoneutra & $970 \mathrm{~b}$ & $700 a$ & $1,39 \mathrm{c}$ & $4032 \mathrm{a}$ & $2284 a$ & 1,76 \\
\hline Quente & $800 \mathrm{c}$ & $555 \mathrm{c}$ & $1,44 b$ & $3100 \mathrm{~b}$ & $1731 \mathrm{~b}$ & 1,79 \\
\hline \multicolumn{7}{|l|}{ Nivel de Selênio* } \\
\hline $\begin{array}{l}\text { Nivel "A" } \\
\text { Nivel "B" }\end{array}$ & $\begin{array}{l}968 \\
972\end{array}$ & $\begin{array}{l}664 \\
668\end{array}$ & $\begin{array}{l}1,55 \\
1,45\end{array}$ & $\begin{array}{l}3929 \\
3918\end{array}$ & $\begin{array}{l}2159 \\
2189\end{array}$ & $\begin{array}{l}1,82 \\
1,79\end{array}$ \\
\hline \multicolumn{7}{|l|}{ Fonte de Selênio } \\
\hline Inorgânico & 919 & 606 & 1,5 & 4045 & 2.172 & 1,79 \\
\hline Orgầnico & 925 & 615 & 1,5 & 4064 & 2.246 & 1,81 \\
\hline \multicolumn{7}{|c|}{ ANOVA, valores de $\mathrm{P}$} \\
\hline Temperatura(TP) & 0,00 & 0,00 & 0,00 & 0,00 & 0,00 & 0,19 \\
\hline Nivel de Se (NS) & 0,73 & 0,06 & 0,28 & 0,47 & 0,50 & 0,51 \\
\hline Fonte de Se (FS) & 0,23 & 0,85 & 0,36 & 0,93 & 0,92 & 0,63 \\
\hline TP $\times N S$ & 0,24 & 0,18 & 0,75 & 0,42 & 0,69 & 0,66 \\
\hline TP $\times F S$ & 0,44 & 0,34 & 0,89 & 0,06 & 0,61 & 0,17 \\
\hline NSXFS & 0,51 & 0,93 & 0,35 & 0,78 & 0,68 & 0,57 \\
\hline $\mathrm{TP} \times \mathrm{NS} \times \mathrm{FS}$ & 0,14 & 0,93 & 0,38 & 0,83 & 0,82 & 0,68 \\
\hline CV\% & 4,49 & 4,04 & 6,0 & 6,75 & 5,63 & 4,2 \\
\hline
\end{tabular}

Médias seguidas de letras distintas em uma mesma coluna, dentro de cada fator, diferem entre si pelo teste de Tukey $(P<0,05)$.

*Nível A: 0,15 mg de Se/kg de ração (1 - 21 dias de idade) e 0,20 mg (21- 42 dias de idade)

Nível B: $0,30 \mathrm{mg}$ de Se/kg de ração (1 - 21 dias de idade) e 0,40 $\mathrm{mg}$ (21- 42 dias de idade)

\section{REFERÊNCIAS}

BECKETT, G.J.; RUSSEL, A.; NICOL, F.; SAHU, P.; WOLF, C.R.;ARTHUR, A.R. Effect of selenium deficiency on hepatic type I 5 'iodothyrinine deiodinase activity and hepatic tyroid hormone levels in rat. Biochemical Journal, Washington, v.282, p.483-487, 1992.

COMBS, G.F. Influences of dietary vitamin $E$ and selenium on the oxidant defense system of the chick. Poultry Science, Champaing, v.60, p.2098-2105, 1981.

FARIA FILHO, D.E. Efeito de dietas com baixo teor protéico, formuladas usando o conceito de proteína ideal, para frangos de corte criados em temperaturas fria, termoneutra e quente. Jaboticabal, 2003, 95f. Dissertação (Mestrado em Zootecnia) - Curso de Pós Graduação em Zootecnia, Universidade Estadual Paulista.

FURLAN, R.L.; MACARI, M. Termoregulação. In.: MACARI, M.; FURLAN, R.L.; GONZALES, E. (Ed.) Fisiologia Aviária aplicada a frangos de corte. 2ed. Jaboticabal: FUNEP, 2002. p.209-230.
HADDAD, E.E.; MASHALY, M.M. Effect of thyroidectomy of immature male chickens on circulatig thyroid hormones and on response to thyroid-stimulating hormones and chronic cold exposure. Poultry Science, Champaing, v.68, p.169176, 1989.

HALPIN, K.M.; BAKER, D.H. Selenium deficiency and transsulfuration in the chick. The Journal of Nutrition, Mesa, v.14, p.606-612, 1984

HAYASHI, K. Roles of thyroid hormone in growth and protein turnover. Animal Science and Technology, Tokyo, v.64, p.938-947, 1993.

HWANG-BO, J.; MURATAMATSU, T.; OKUMURA, J. Relative of triiodo-thyronine and of thyroxine for inducing oxygen consumption in young chicks. Poultry Science, Champaing, v.69, p.1027-1029, 1990.

JIANHUA, H.; OHTSUKA, A.; HAYASHI, K. Selenium influences growth via thyroid hormone status in broiler chickens. British Poultry Science, London, v.84, p.727-732, 2000. 
LEESON, S. Nutritional considerations of poultry during heat stress. World's Poultry Science, Baltimore, v.42, p.69-81, 1986.

LONGO, F.A. Estudo do metabolismo energético e do crescimento em frangos de corte. Jaboticabal, 2000. 76f. Dissertação (Mestrado em Zootecnia) - Curso de Pós Graduação em Zootecnia, Universidade Estadual Paulista.

MELTZER, A. Aclimatization to ambient temperature and its nutritional consequences.

Recebido para publicação: 25/01/2005

Aprovado:

$11 / 04 / 2005$
World's Poultry Science, Baltimore, v.43, n.1, p.33-45, 1987.

MOREIRA, J.; SANTOS, C.D.; ABREU, C.M.P.; BERTECHINI, A.G.; CARDOSO, M.G. Efeito de fonte e níveis da glutationa peroxidase e no desempenho de frangos de corte. Ciência Agrotécnica, Lavras, v.25, p.645649, 2001.

SAS INSTITUTE (Cary, United States). SAS ${ }^{\circledR}$ User's Guide: Statistics. Cary, 2002. 